\title{
A Low-Complexity Memoryless Model for Envelope Tracking RF Power Amplifiers
}

\author{
G. Montoro, P. L. Gilabert \\ Dept. of Signal Theory and Communications \\ Universitat Politècnica de Catalunya (UPC-BarcelonaTech) \\ Campus del Baix Llobregat \\ Castelldefels (Spain) \\ gabriel.montoro@upc.edu
}

\author{
J. A. Garcia, M. N. Ruiz, R. Marante \\ Dept. of Communications Engineering \\ Universidad de Cantabria (UC) \\ Santander (Spain)
}

\begin{abstract}
This paper presents a new kind of behavioral model structure for characterizing static nonlinearities in dynamically supplied RF power amplifiers. The proposed architecture is based on the combination of several basic cells and it is focused to simplify the real-time design and implementation of adaptive shaping functions devoted to improve the efficiency in systems controlled by digital signal processors with tight computational capabilities, such is the case of handsets and small-cells.
\end{abstract}

Keywords- envelope tracking modeling, shaping function, amplifiers linearity and efficiency

\section{INTRODUCTION}

High speed data rates and high spectral efficiency required in modern communication technologies are pushing new communication standards to transmit signals based on linear modulations schemes and exhibiting fast envelope variations. This kind of signals forces the operation of the power amplifier (PA) at high back-off levels to avoid distortions on the linear modulations, degrading the PA efficiency. Topologies based on dynamic power supply, such as Envelope Tracking (ET) [1], have been actively investigated as possible solutions to achieve linear and high efficient amplification.

One of the main issues regarding the efficiency achieved by envelope tracking power amplifiers regards the modeling and compensation of the mismatches inherent to the degradation due to the dynamic supply [2]. Moreover, the bandwidth of the signal's envelope is several times (theoretically is infinite) the bandwidth of the baseband modulated signal. That means that sometimes it is necessary to use, as a supply signal, a modified envelope not univocally related to the original envelope. In this case, the nonlinearities are both dependent on the amplifiers signal and on the signal used to supply, and both variables must be considered in the model. For instance, this is the case when a slower version of the original signal's envelope is used to perform drain modulation [3] [4]. This generates additional nonlinear distortion at the RF PA's output.

This paper is oriented at finding a simple model able to characterize the behavior of memoryless dynamically supplied RF power amplifiers, as is the case of the well-known envelope tracking topology. The idea is to use this simplified model for designing and implementing adaptive shaping functions. Each time the model is updated means that the shaping function will be fulfilled with new values.

\section{ET BEHAVIORAL MODELING}

\section{A. ET RF Power Amplifier Structure}

The structure of an ET amplifier is shown in Fig. 1. The baseband signal to be amplified is $x_{R}$, the real envelope of $x_{R}$ is $E$ and this is modified by an envelope shaping function [5] [6] in order to obtain $E_{S}$. The supply signal $E_{S}$ is amplified by the envelope amplifier (EA) and then the resulting $E_{D}$ is used to feed the RF PA (usually a class A or AB topology). Fig. 1 shows the baseband signal processing associated to this topology, while all the subsystems necessary for moving the baseband signal to/from the RF (in band-pass domain) are obviated. From a behavioral point of view, we can consider the overall system as a black-box with baseband inputs and outputs. In order to improve the linearity, a digital predistortion (DPD) block can be included in the baseband path.

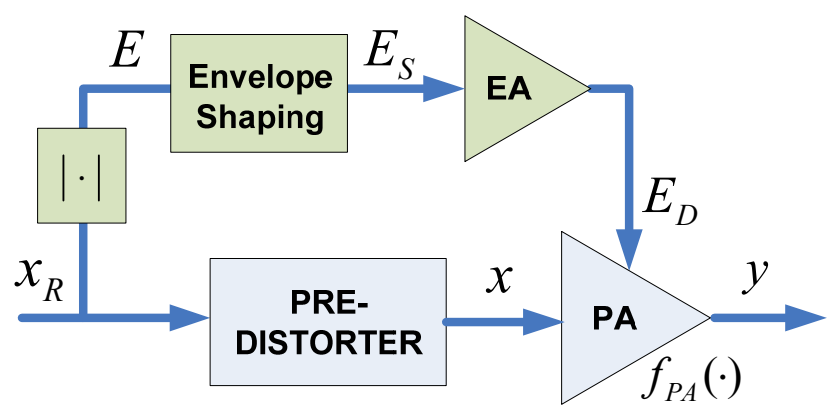

Fig. 1. Conventional structure of an Envelope Tracking amplification topology.

\section{B. ET Modeling}

The mathematical behavioral model of the ET system must consider both the dependence of the baseband input signal $x_{R}$ and the dependence of the signal used for supplying the PA, 
$E_{S}$, as described in the following equation in the discrete time domain [4]

$$
y(n)=\sum_{p=0}^{P} \sum_{q=0}^{Q} \gamma_{p q}|x(n)|^{p} E_{S}^{q}(n) x(n)
$$

with $x$ and $y$ being the baseband PA input and output signals, respectively, and $E_{S}$ the PA supply. This notation will be maintained from now onwards along the paper.

\section{LOW-COMPLEXITY MEMORYLESS ET PA BEHAVIORAL MODEL}

In some cases the model indicated in the previous section, see (1), can be computationally too intensive to be used for PA characterization, and for DPD or envelope shaping implementation in small digital signal processors, as is the case of handset and small-cell PA applications. Doing a truncation of the series expansion implies a complexity simplification, but this means degradation in the capabilities of the model to fit the real PA.

\section{A. Proposed Low-Complexity ET Model}

The proposed model structure consists in the parallel connection of several basic cells, each one composed by the series connection of two basic blocks. One of them is devoted to model the conventional PA static behavior, as corresponds to an AM-AM and AM-PM characterization. The second one is devoted to characterize the dynamic supply effects. These cells and blocks interconnections are shown in Fig. 2, and mathematically are described by the following equation

$$
y=\sum_{i=1}^{N} f_{i}(|x|) g_{i}\left(E_{s}\right) x
$$

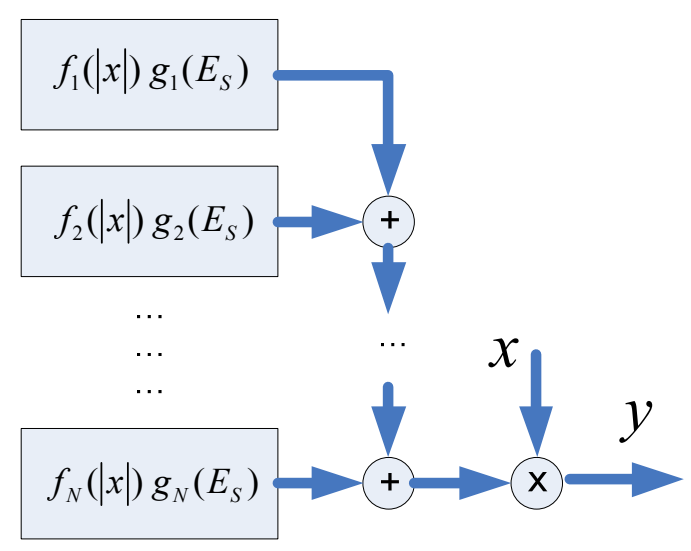

Fig. 2. Interconexion of $\mathrm{N}$ basic modeling cells.

where the signal $x$ and $y$ correspond to the baseband PA input and output, respectively. Moreover, each one of the static nonlinear functions $f_{i}(\cdot)$ and $g_{i}(\cdot)$ can be expressed as

$$
f_{i}(|x|)=\sum_{p=0}^{P} \alpha_{i p}|x|^{p} ; \quad g_{i}\left(E_{S}\right)=\sum_{q=0}^{Q} \beta_{i q} E_{S}^{q}
$$

Each one of the $f_{i}(\cdot)$ and $g_{i}(\cdot)$ polynomial expressions can be implemented in look-at tables (LUT). That means to spend memory resources in the baseband digital processor but saving the real-time implementation of arithmetical operations (sums and products) [7]. However, if the model expansion has few terms then the real-time arithmetical implementation of the truncated expansion can be considered. For instance, a third order expansion of the supply dependent term $g_{i}\left(E_{S}\right)$ looks as

$$
g_{i}\left(E_{S}\right)=\beta_{i 0}+\beta_{i 1} E_{S}+\beta_{i 2} E_{S}^{2}+\beta_{i 3} E_{S}^{3}
$$

Usually, in ET amplifiers the nonlinearities can be bounded up to the third order (term $E_{S}^{3}$ ), therefore the expansion described in (4) is enough. Fig. 3 shows the connection of the two gain blocks that composes a basic cell.

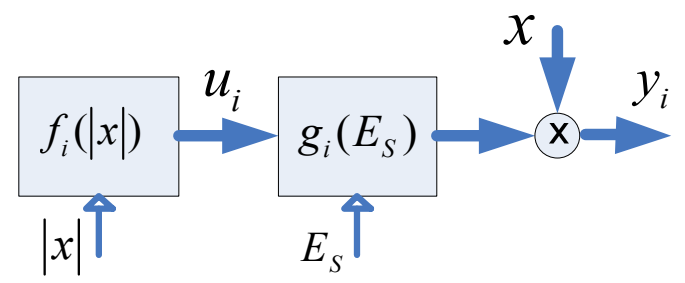

Fig. 3. Basic cell of the proposed model.

The signals $u_{i}=f_{i}(|x|)$ are defined as intermediate signals. Then, next step is to establish a technique for updating the coefficients of the model described by (2) and (3). In order to find the best LS (least squares) estimation of the coefficients the following methods, proposed in literature for solving similar estimation problems, are considered: a) Decomposition [8], b) Intermediate signal technique [9], c) Iterative method [10]. Therefore, these three estimation methods could be applied to identify the coefficients of the basic cell structure shown in Fig. 3.

Besides, in order to further simplify the computational cost of the parameters estimation, the idea is to estimate each one of the basic cells separately. For doing this, in this case the best option is to do several iterative LS estimations (option c), similar to the method described in [10]. Therefore, each block of each cell will be estimated separately by using the residual error and doing a local conventional LS fit. So, each one of the two terms of a cell, $f_{i}(\cdot)$ and $g_{i}(\cdot)$, are calculated separately and sequentially. The advantage of doing this is the distribution of the computational cost of each one of the local LS parameters calculation, in relation to do a full LS of the whole parameters, as is the case of solving the LS parameters in the overall model described by (1). Doing several local LS fittings is useful when considering an adaptive model in order to 
implement an adaptive digital predistorter (DPD) or an adaptive shaping function design. This will be shown in the next section.

\section{B. Creating Shaping Functions}

For instance, in the following example we want to follow an isogain strategy [6], that is, supplying the PA with the signal $E_{S}$ that induces a constant gain and thus minimizes the gain distortion. In this case, if we consider a model with 2 basic cells, the strategy consists in obtaining the best $E_{S}$ that minimizes the following cost function

$$
J_{\text {error }}\left(E_{S}\right)=\left|u_{1} g_{1}\left(E_{S}\right)+u_{2} g_{2}\left(E_{S}\right)-g_{o}\right|
$$

where $g_{0}$ corresponds to the desired gain. After obtaining $u_{1}=f_{1}(|x|), \quad u_{2}=f_{2}(|x|), \quad g_{1}\left(E_{S}\right)$ and $g_{2}\left(E_{S}\right)$, by solving the corresponding local LS fittings, next step is to find for each $|x|$ the $E_{S}$ supply that minimizes $J_{\text {error }}\left(E_{S}\right)$. This can be done effectively exciting de $g_{i}(\cdot)$ terms by a ramp signal including all the allowed supply values and then choosing the best one and storing it in the final shaping function LUT.

\section{EXPERIMENTAL RESULTS}

\section{A. Experimental test-bench}

The experimental test-bench is shown in Fig. 4. For testing purposes, we used a broadband high efficiency continuousmode class $\mathrm{J}$ power amplifier at $950 \mathrm{MHz}$, based on the CGH35030F packaged GaN HEMT from Cree Inc.

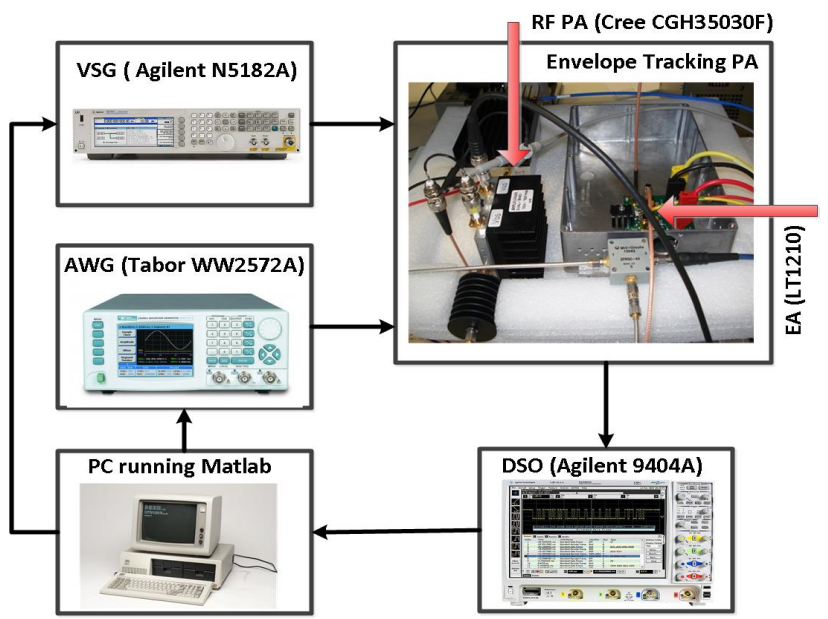

Fig. 4. Experimental test-bench.

The signal generation and measurement equipment consist of: Agilent MXG N5182A RF vector generator, Tabor WW2572A arbitrary wave generator, and an Agilent Infinium DSO9404A oscilloscope for capturing the signals. The overall system is controlled by a PC running Matlab. We used as the EA the high-speed ( $35 \mathrm{MHz}$ bandwidth and $900 \mathrm{~V} / \mu$ s slew-rate at $\mathrm{Av}=2$ and $10 \Omega$ load) high-current (1.1 A) Linear
Technology IC LT1210. For the sake of simplicity we have considered the slightly efficient IC LT1210 as the envelope driver because the scope of this work is to prove the performance of the proposed modeling and shaping design method.

\section{B. Results: PA Modeling and dynamic supply design}

By using the proposed structure indicated in the previous section, the following PA characterization results are obtained. As excitation, a LTE signal of $5 \mathrm{MHz}$ bandwidth $(8.3 \mathrm{~dB}$ of PAPR) and a 2TONES signal (1 MHz of separation between tones) have been used.

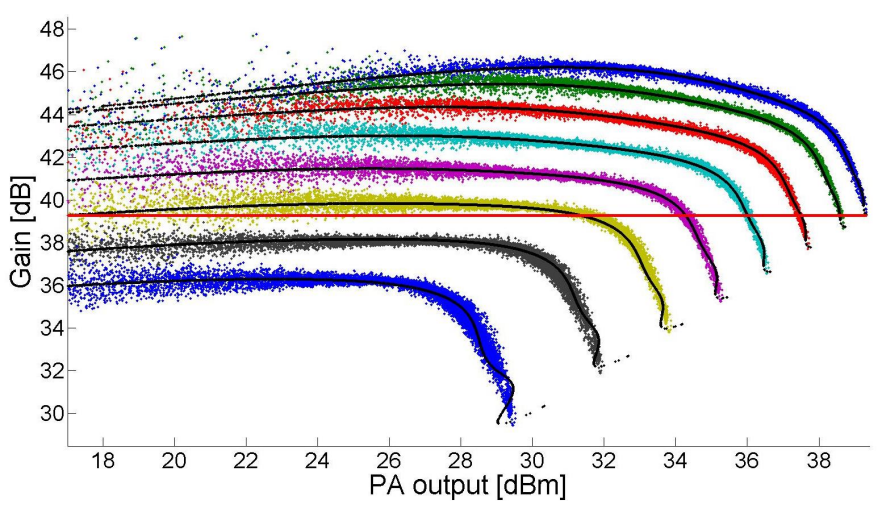

Fig. 5. Captured data and Least Squares (LS) estimation results of the PA response when aplying a LTE signal of $5 \mathrm{MHz}$ BW. A total of 8 supply levels have been tested (from $28 \mathrm{~V}$ to $8.4 \mathrm{~V}$ ).

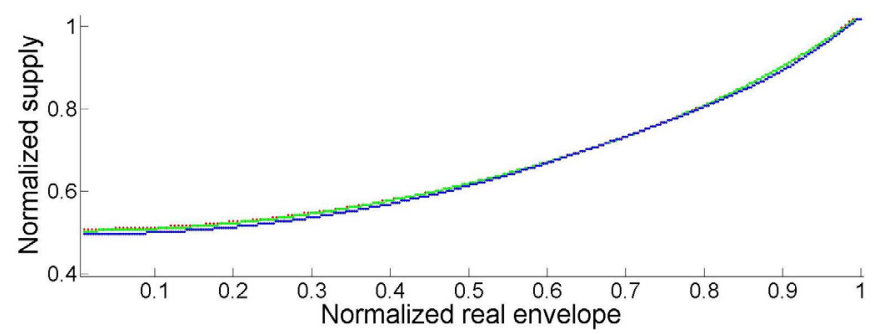

Fig. 6. Resulting shaping functions (blue: proposed method with 1 cell, green: 2 cells, red: series expansion - the plots are almost overlapped). In all cases have been obtained from the 2 TONES signal training.

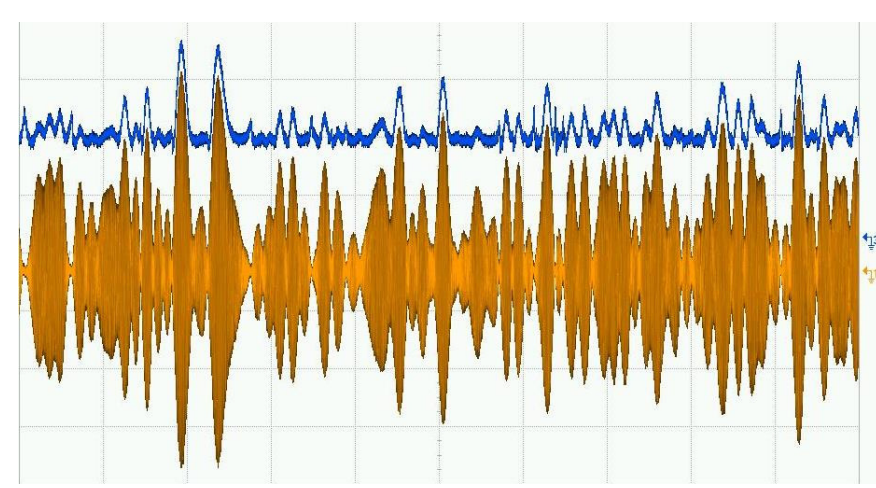

Fig. 7. Oscilloscope captures of the RF and the constant gain dynamic supply. 
The PA responses and LS estimation for a set of supply voltages are plot in Fig. 5. At each test the DC supply is changed, going from $28 \mathrm{~V}$ to $8.4 \mathrm{~V}$. Moreover Fig. 6 shows the resulting constant gain, or isogain [6], shaping function obtained when the proposed model is used (for 1 and 2 cells) and when a series expansion is used. Fig. 7 shows the real and the constant gain envelopes.

TABLE I: Modeling results

\begin{tabular}{|c|c|c|c|}
\hline & \multirow{2}{*}{\begin{tabular}{l}
\multicolumn{1}{c}{ LS } \\
Series \\
expansion
\end{tabular}} & \multicolumn{2}{|c|}{ Proposed model } \\
\hline & & 1 basic cell & 2 basic cells \\
\hline $\begin{array}{l}\text { NMSE between } \\
\text { estimated and } \\
\text { real PA complex } \\
\text { outputs. Cases: } \\
\text { LTE5MHz/ } \\
\text { 2TONES } \\
\text { [dB] }\end{array}$ & $\begin{array}{l}-30.28 / \\
-41.43\end{array}$ & $\begin{array}{c}-25.37 / \\
-30.4\end{array}$ & $\begin{array}{l}-30.33 / \\
-40.98\end{array}$ \\
\hline $\begin{array}{l}\text { \# of parameters } \\
\text { used in the LS } \\
\text { fittings }\end{array}$ & $\begin{array}{l}\text { Order } 6 \text { for } \\
\text { the }|x| \text { term } \\
\text { and order } 4 \\
\text { for the Es. } \\
\text { A total of } 35 \\
\text { terms. }\end{array}$ & $\begin{array}{l}2 \text { independent } \\
\text { LS fittings: } 1 \\
\text { of order } 6(|x|) \\
\text { and } 1 \text { of order } \\
4 \text { (Es) }\end{array}$ & $\begin{array}{l}4 \text { independent } \\
\text { LS fittings: } 2 \\
\text { of order } 6(|x|) \\
\text { and } 2 \text { of order } \\
4(\text { Es) }\end{array}$ \\
\hline
\end{tabular}

TABLE II: Dynamic supply results for LTE (BW of $5 \mathrm{MHz}$ )

\begin{tabular}{|l|c|l|c|c|}
\hline \multirow{2}{*}{} & \multicolumn{2}{|c|}{ Shaping technique } & \multicolumn{1}{c|}{ DC } & DC \\
\cline { 2 - 5 } & $\begin{array}{l}\text { Real } \\
\text { envelope }\end{array}$ & $\begin{array}{l}\text { Constant } \\
\text { gain } \\
\text { (designed } \\
\text { from the } \\
\text { model } \\
\text { trained } \\
\text { with } \\
\text { 2TONES) }\end{array}$ & $\begin{array}{l}\text { Supply } \\
\text { voltage } \\
\mathbf{2 8} \text { V }\end{array}$ & $\begin{array}{l}\text { Supply } \\
\text { voltage } \\
\mathbf{2 8 ~ V} \\
+ \text { output } \\
\text { backoff }\end{array}$ \\
\hline $\begin{array}{l}\text { Mean output } \\
\text { power } \\
\text { [W] }\end{array}$ & 0.85 & 1.38 & 3.52 & 1.14 \\
\hline $\begin{array}{l}\text { NMSEs } \\
\text { (complex/ } \\
\text { modulus) } \\
\text { [dB] }\end{array}$ & $-14.12 /$ & $-23.7 /$ & $-14.68 /$ & $-21.92 /$ \\
\hline $\begin{array}{l}\text { ACLR } \\
\text { (L/U) } \\
\text { [dBc] }\end{array}$ & $-21.41 /$ & $-31.9 /$ & $-22.8 /$ & $-30.81 /$ \\
\hline $\begin{array}{l}\text { Drain } \\
\text { consumption } \\
\text { [W] }\end{array}$ & 1.72 & 2.96 & 7.83 & -30.48 \\
\hline $\begin{array}{l}\text { Drain } \\
\text { efficiency } \\
\text { [\%] }\end{array}$ & 49.42 & 46.62 & 44.95 & 27.14 \\
\hline
\end{tabular}

Table I shows a comparison of the implementation of the proposed modeling method in relation to the series expansion model described in (1). Table II shows performance results for the constant gain design (target mean output power of $31 \mathrm{dBm}$, $1.25 \mathrm{~W}$ ), and it is compared to the shaping with real envelope and to some DC supply cases. As shown in Table II, in the case of the constant gain shaping the difference between the NMSE of the complex signals and the NMSE of the modulus is of around $7 \mathrm{~dB}$ : this means the presence of residual AM-PM distortion, that could be compensated by means of an IQ DPD.

\section{CONCLUSION}

The proposed ET behavioral model allows the distribution of the computational cost of solving the necessary LS estimation for obtaining the coefficients of the model. Moreover, it is suitable for implementing adaptive shaping functions in the computationally limited digital signal processors used in handsets and other RF devices that incorporates low and medium power PAs.

\section{ACKNOWLEDGMENT}

This work was supported by the Spanish Ministry MINECO under projects TEC2011-29126-C03 (-01 and -02).

\section{REFERENCES}

[1] D. F. Kimball, J. Jeong, C. Hsia, P. Draxler, S. Lanfranco, W. Nagy, K. Linthicum, L. E. Larson and P. M. Asbeck., "High-efficiency envelopetracking WCDMA base-station amplifier using GaN HFETs," IEEE Trans. on Microw. Theory and Tech., vol. 54, pp. 3848 - 3856, Nov. 2006.

[2] P. J. Draxler, "Behavioral modelling of nonlinearities and memory effects in power amplifiers," PhD Thesis, University of California, San Diego, USA, 2013.

[3] P. L. Gilabert, G. Montoro, "Look-Up Table implementation of a slow envelope dependent digital predistorter for envelope tracking power amplifiers," IEEE Microwave and Wireless Components Letters, vol. 22, pp. 97-99, Feb. 2012.

[4] G. Montoro, P. L. Gilabert, et al., "Digital predistortion of envelope tracking amplifiers driven by slew-rate limited envelopes," IEEE MTT-S Int. Microw. Symp. (IMS'11), June. 2011, Baltimore, USA.

[5] N. T. Giovannelli, A. Cidronali and G. Manes, "Efficiency and linearity enhancements with envelope shaping control in wideband envelope tracking GaAs PA," 2011 Workshop on INMMIC, April 2011.

[6] G. Wimpenny and J. Hendy, "DPD and envelope tracking", Workshop on Holistic Approach to Transceiver Architectures and Technologies to Femto/Pico Cell Based Communication Systems, IEEE MTT-S Int. Microw. Symp. (IMS'13), June 2013, Seattle, USA.

[7] P. L. Gilabert, A. Cesari, G. Montoro et al. "Multi Look-Up Table FPGA implementation of an adaptive digital predistorter for linearizing RF power amplifiers with memory effects," IEEE Trans. on Microw. Theory and Tech., vol. 56, pp. 372 - 384 , Feb. 2008.

[8] E. W. Bai, "An optimal two stage identification algorithm for Hammerstein-Wiener nonlinear systems," Proc. American Contr. Conf., Philadelphia, Pennsylvania, June, 1998, 2756-2760.

[9] A. Hagenblad, "Aspects of the identification of Wiener models," $\mathrm{PhD}$ Thesis Division of Automatic Control, Department of Electrical Engineering Link"opings Universitet, Sweden, 1999.

[10] K. S. Narendra and P. G. Gallman, "An iterative method for the identification of nonlinear systems using a Hammerstein model," IEEE Trans. Automat. Contr., vol. AC-11, pp. 546-550, July 1966. 